\title{
Coronary fat content evaluated by morphometry in patients with severe atherosclerosis has no relation with serum lipid levels
}

D.J. Braz Jr., P.S. Gutierrez and P.L. da Luz
Unidade de Ateroscleroses e Laboratório de Patologia, Instituto do Coração, Faculdade de Medicina, Universidade de São Paulo, São Paulo, SP, Brasil

\section{Correspondence}

P.L. da Luz

Av. Dr. Enéas C. Aguiar, 44

05403-000 São Paulo, SP

Brasil

Fax: +55-11-3069-5447

E-mail: daluzp@incor.usp.br

....................

Publication supported by FAPESP.

Received September 12, 2005 Accepted October 6, 2006

\begin{abstract}
The relationship between lipid serum levels and coronary atherosclerotic plaque fat content was studied in 51 necropsy patients. Serum lipids were measured by standard techniques, during life, in the absence of lipidlowering drugs. Intima, intimal fat and media areas were measured using a computerized system in cryosections of the odd segments of the right, anterior descending and circumflex coronary arteries stained with SudanIV. Mean intimal and lipid areas were $5.74 \pm 1.98$ and $1.22 \pm 0.55 \mathrm{~mm}^{2}$ $(22.12 \pm 8.48 \%)$ in 26 cases with high cholesterol ( $\geq 200 \mathrm{mg} / \mathrm{dL})$ and 4.98 \pm 1.94 and $1.16 \pm 0.66 \mathrm{~mm}^{2}(22.75 \pm 9.06 \%)$ in 25 cases with normal cholesterol $(<200 \mathrm{mg} / \mathrm{dL}$; P $>0.05)$. Patients with high levels of lowdensity lipoprotein $(\geq 130 \mathrm{mg} / \mathrm{dL}, \mathrm{N}=15)$ had a higher intima/media area ratio than those with normal levels of low-density lipoprotein $(<130 \mathrm{mg}$ / $\mathrm{dL}, \mathrm{N}=13, \mathrm{P}<0.01)$. No significant difference in the morphometrical variables was found in groups with high or low serum levels of triglycerides ( $\geq 200 \mathrm{mg} / \mathrm{dL}, \mathrm{N}=13 v s<200 \mathrm{mg} / \mathrm{dL}, \mathrm{N}=36$ ) or high-density lipoprotein ( $\geq 35 \mathrm{mg} / \mathrm{dL}, \mathrm{N}=11 \mathrm{vs}<35 \mathrm{mg} / \mathrm{dL}, \mathrm{N}=17)$. The association between the morphological measurements and serum levels of cholesterol, its fractions, and triglycerides was also tested and the correlation coefficients were low. Although high cholesterol is a risk factor, we show here that in patients with severe atherosclerosis blood cholesterol and triglyceride levels seem to have little influence on coronary lipid content, indicating that other factors may contribute to arterial lipid deposition and plaque formation.
\end{abstract}

\section{Introduction}

The development of atherosclerosis has been consistently linked to risk factors such as high cholesterol, hypertension, smoking, and diabetes (1). Aggressive lipid-lowering therapy causes significant reductions in coronary events, global and cardiovascular mor-
Key words

- Coronary arteriosclerosis

- Cholesterol

- Image cytometry tality, and surgical revascularization, thus proving the importance of hypercholesterolemia (2). However, an important fraction of the patients with coronary artery disease (CAD) do not have high-serum lipid levels and indeed may not have any traditional risk factors (3), suggesting that additional factors contribute to lesion formation. This fact also 
poses a significant management problem, since stabilization, slowed progression or even regression of atherosclerotic lesions have primarily been described in hypercholesterolemic individuals (4).

Several investigators have examined the morphologic characteristics of coronary arteries in patients with CAD who died with different clinical syndromes. In general the arteries were found to be diffusely affected, but morphologic appearance and quantitative composition of plaques varied considerably. Plaques with a large amount of necrotic and lipidic core are considered to be more vulnerable (prone to rupture and consequently thrombosis) and linked to acute coronary events; conversely, plaques with a predominance of the fibrous cap are more related to stable coronary syndromes $(5,6)$. The relationship between blood cholesterol and triglyceride levels and the actual deposition of fat in the coronary arteries has not been assessed in spite of the critical importance this knowledge would have for both the understanding of the pathophysiology of atherosclerosis and patient treatment. Thus, the objective of the present study was to determine the relationship between plasma lipid and plaque fat content.

\section{Patients and Methods}

The study was approved by the Scientific and Ethics Committee, Heart Institute (InCor), University of São Paulo Medical School.

\section{Selection of cases, clinical and biochemical data}

The autopsy records of the Incor, University of São Paulo Medical School, São Paulo, SP, Brazil, between 1993 and 1998, were surveyed for patients coded as having atherosclerosis. Medical data were collected and 50 patients who had their plasma cholesterol measured during life, in the absence of lipid-lowering drugs, were selected. One heart of a transplant recipient was also included. Forty-four cases had recent and/or scarred myocardial infarction; the remaining ones had died due to other cardiac causes, mostly complications of surgical coronary revascularization. In 49 of the patients, triglycerides were also quantified, and very low-density lipoprotein (VLDL) was calculated by the formula: triglycerides/5. In 28 cases, high-density lipoprotein (HDL) and low-density lipoprotein (LDL) were also measured. Total cholesterol, HDL and triglycerides had been measured using enzymatic assays (CHOD-PAP, MSD) and LDL was calculated by the Friedewald formula (7). Glucose was measured by an enzymatic method using hexokinase, available from Cobas Integra 700 (Roche Diagnostic Systems, Inc., Branchburg, NJ, USA). Additional major risk factors had also been recorded, such as smoking, diabetes mellitus and hypertension.

\section{Specimens, slide preparation and morphometric quantification}

The hearts were fixed in formalin. The left anterior interventricular (anterior descending, LAD) and left circumflex (Cx) branches of the left coronary artery and the right coronary artery were excised, decalcified and cut transversely at $1-\mathrm{cm}$ intervals. The odd centimeters were studied. Two to 5 sections were analyzed in each vessel, for a total of 10 to 12 in each case. The arterial segments were labeled sequentially from their aortic origin; after transverse cutting, the segments were frozen. Fifteen-micrometer thick sections were cut and stained with Sudan-IV to reveal fat. Of 528 sections initially examined, 24 were excluded because the sections included branch points or artifacts; thus, 504 sections remained for examination.

The following areas were quantified by planimetry with Quantimet-500 optical system (Leica Microsystems, Wetzlar, Hessen, 
Germany) using $2.5 \mathrm{X}$ lens magnification: intimal area (total area enclosed by the internal elastic membrane minus the lumen area), media (total area enclosed by the two elastic membranes) and fat area (area stained red in the intima) (Figure 1). Additionally, the ratio between the intimal and medial areas and the proportion of fat composing the intima (ratio between intimal fat and total intimal area) were calculated. The mean value of each of these variables was calculated for each coronary branch; for each case, the values considered were the mean of the results of the three branches.

\section{Statistical analysis}

Data were analyzed statistically by two approaches: 1) comparison of morphometric findings between groups of patients with low and high levels of cholesterol $(<200 \mathrm{vs}$ $\geq 200 \mathrm{mg} / \mathrm{dL}$ ), triglycerides $(<200 v s \geq 200$ $\mathrm{mg} / \mathrm{dL}$ ), HDL ( $<35 v s \geq 35 \mathrm{mg} / \mathrm{dL}$ ), and LDL $(<130 v s \geq 130 \mathrm{mg} / \mathrm{dL})$. The $t$-test was applied if the data distribution was normal, and the Mann-Whitney test was applied if distribution was not. 2) Evaluation of the association between the morphological measurements and biochemical serum levels of cholesterol, its fractions, and triglycerides, including all patients in a single group. The Pearson correlation test was used.

Since VLDL $=$ triglycerides $/ 5$, statistical analysis would be identical for these two variables, and thus only triglyceride levels were tested.

The level of significance was established at $5 \%$ and the SigmaStat ${ }^{\circledR}$ system (SPSS Inc., Chicago, IL, USA) was used for all statistical calculations.

\section{Results}

\section{Comparison between patients with high and} low cholesterol levels

Clinical data. The clinical features of patients classified according to serum cholesterol levels are summarized in Table 1. The mean cholesterol level was $234.05 \pm$ $33.83 \mathrm{mg} \%$ for the group with high serum levels ( $\mathrm{N}=26)$ and $162.71 \pm 26.88 \mathrm{mg} \%$ for the other group $(\mathrm{N}=25)$. Age ranged from 41 to 81 years (mean $61.73 \pm 11.79$, median $63.5)$ in the group with high cholesterol and from 42 to 83 years (mean $66.60 \pm 9.77$, median 67) in the group with normal cholesterol. There was no significant difference between the groups with low and high cholesterol levels concerning age, gender, smoking habit, diabetes, and hypertension, with a high incidence of the last condition in both of them. The blood concentrations of HDL $(29.40 \pm 9.33$ vs $35.30 \pm 11.25 \mathrm{mg} / \mathrm{dL}, \mathrm{P}=$

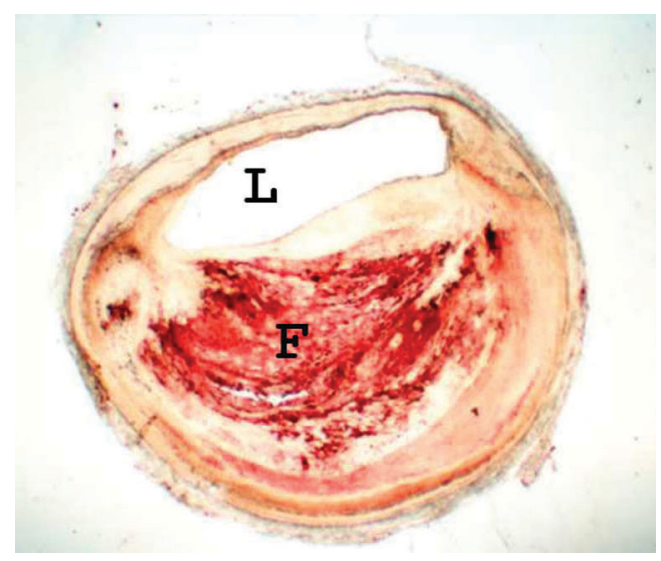

Figure 1. Transverse frozen section of a coronary artery. The red image corresponds to the fat-positive areas $(F) . L=$ arterial lumen. Sudan-IV staining, objective magnification $=1.25 \mathrm{X}$.
Table 1. Gender, age, and presence of systemic arterial hypertension (SAH), diabetes mellitus or smoking habit in patients from the normocholesterolemic and hypercholesterolemic groups studied.

$\begin{array}{cc}\begin{array}{c}\text { Normocholesterolemic } \\ \text { (cholesterol }<200 \mathrm{mg} \%) \\ (\mathrm{N}=25)\end{array} & \begin{array}{r}\text { Hypercholesterole } \\ \text { (cholesterol } \geq 200 \\ (\mathrm{~N}=26)\end{array} \\ 61.73 \pm 11.79 & 66.60 \pm 9.77 \\ 13(52 \%) & 17(65.4 \%) \\ 21 & 20 \\ 7 & 12 \\ 6 & 3\end{array}$

Age data are reported as means \pm SD and all other data as the number of patients. Age was analyzed by the $t$-test, gender and diabetes by the chi-square test, and SAH and smoking habit by the Fisher exact test. There were no statistically significant differences between groups. 
$0.15)$ and triglycerides $(140.47 \pm 70.15 v s$ $183.48 \pm 90.44 \mathrm{mg} / \mathrm{dL} ; \mathrm{P}=0.07$ ) were comparable, but LDL (103.20 29.95 vs 156.06 $\pm 25.54 \mathrm{mg} / \mathrm{dL} ; \mathrm{P}<0.01$ ) was lower in the group with low cholesterol levels.

Morphometric results. Measurements of intima, fat area, proportion of fat, media or intima/media ratio were similar for all patients regardless of their total cholesterol, triglycerides, and HDL ( $\mathrm{P}>0.05$; Table 2). The only significant difference found was that patients with high LDL cholesterol fractions had a higher intima/media area ratio
$(3.85 \pm 1.32$ vs $2.43 \pm 1.10, \mathrm{P}<0.01)$.

\section{Relationship between measured areas and} serum levels of cholesterol and triglycerides

The correlation coefficients (r) between serum levels of total cholesterol, triglycerides, HDL, and LDL, and measured areas of coronary artery intimal area, fat area and fat percentage in the intima are reported in Table 3. The correlation between LDL and the intima/ media area ratio was significant $(\mathrm{P}<0.01, \mathrm{r}=$ $0.55)$, possibly due to the negative association

Table 2. Intimal area, fat area, percent fat in intima, media area, and intima/media area ratio in groups with high and normal serum cholesterol, triglyceride, high-density lipoprotein (HDL), and low-density lipoprotein (LDL) levels.

\begin{tabular}{|c|c|c|c|c|c|c|}
\hline & $\mathrm{N}$ & $\begin{array}{l}\text { Intima area } \\
\left(\mathrm{mm}^{2}\right)\end{array}$ & $\begin{array}{c}\text { Fat area } \\
\left(\mathrm{mm}^{2}\right)\end{array}$ & $\%$ Fat & $\begin{array}{l}\text { Media area } \\
\left(\left(\mathrm{mm}^{2}\right)\right.\end{array}$ & $\begin{array}{c}\text { Intima/media } \\
\text { area ratio }\end{array}$ \\
\hline \multicolumn{7}{|l|}{ Cholesterol } \\
\hline$\geq 200 \mathrm{mg} / \mathrm{dL}$ & 26 & $5.74 \pm 1.98$ & $1.22 \pm 0.55$ & $22.12 \pm 8.48$ & $1.78 \pm 0.69$ & $3.49 \pm 1.34$ \\
\hline$<200 \mathrm{mg} / \mathrm{dL}$ & 25 & $4.98 \pm 1.94$ & $1.16 \pm 0.66$ & $22.75 \pm 9.06$ & $1.88 \pm 0.56$ & $2.84 \pm 1.27$ \\
\hline \multicolumn{7}{|l|}{ Triglycerides } \\
\hline$\geq 200 \mathrm{mg} / \mathrm{dL}$ & 13 & $5.51 \pm 2.21$ & $1.47 \pm 0.71$ & $27.18 \pm 9.09$ & $2.03 \pm 0.69$ & $2.88 \pm 1.30$ \\
\hline$<200 \mathrm{mg} / \mathrm{dL}$ & 36 & $5.32 \pm 1.98$ & $1.08 \pm 0.55$ & $20.54 \pm 8.17$ & $1.77 \pm 0.60$ & $3.25 \pm 1.36$ \\
\hline \multicolumn{7}{|l|}{$\mathrm{HDL}$} \\
\hline$<35 \mathrm{mg} / \mathrm{dL}$ & 17 & $5.84 \pm 2.12$ & $1.25 \pm 0.72$ & $20.41 \pm 8.24$ & $1.76 \pm 0.51$ & $3.55 \pm 1.58$ \\
\hline$\geq 35 \mathrm{mg} / \mathrm{dL}$ & 11 & $5.69 \pm 2.13$ & $1.27 \pm 0.73$ & $21.74 \pm 8.70$ & $2.09 \pm 0.70$ & $2.96 \pm 1.28$ \\
\hline \multicolumn{7}{|l|}{ LDL } \\
\hline$\geq 130 \mathrm{mg} / \mathrm{dL}$ & 15 & $6.33 \pm 1.75$ & $1.49 \pm 0.68$ & $23.11 \pm 7.32$ & $1.75 \pm 0.60$ & $3.85 \pm 1.32$ \\
\hline$<130 \mathrm{mg} / \mathrm{dL}$ & 13 & $5.08 \pm 2.32$ & $1.01 \pm 0.70$ & $19.03 \pm 9.63$ & $2.20 \pm 0.63$ & $2.43 \pm 1.10^{*}$ \\
\hline
\end{tabular}

Data are reported as means \pm SEM and were compared between serum groups with two levels by either the $t$-test or Mann-Whitney test. $\mathrm{P} \leq 0.05$ is indicated by asterisks.

Table 3. Correlation coefficients between serum levels of total cholesterol, triglycerides, high-density lipoprotein (HDL), and low-density lipoprotein (LDL), and measured areas of coronary artery intima area, fat area, percent fat in intima, media area, and intima/media area ratio.

\begin{tabular}{lccccc}
\hline & $\begin{array}{c}\text { Intima area } \\
\left(\mathrm{mm}^{2}\right)\end{array}$ & $\begin{array}{c}\text { Fat area } \\
\left(\mathrm{mm}^{2}\right)\end{array}$ & \% Fat & $\begin{array}{c}\text { Media area } \\
\left(\mathrm{mm}^{2}\right)\end{array}$ & $\begin{array}{c}\text { Intima/media } \\
\text { area ratio }\end{array}$ \\
\hline Cholesterol & 0.16 & 0.15 & 0.16 & -0.25 & $0.32^{*}$ \\
Triglycerides & 0.08 & $0.28^{*}$ & $0.35^{*}$ & 0.18 & -0.11 \\
HDL & 0.14 & 0.10 & 0.04 & -0.18 & 0.23 \\
LDL & 0.28 & 0.23 & 0.16 & $-0.44^{*}$ & $0.55^{*}$ \\
\hline
\end{tabular}

Correlation coefficients were determined by the Pearson test.

${ }^{*}$ Probability of $\alpha$ error $(\mathrm{P} \leq 0.05)$. 
between LDL and medial area $(\mathrm{P}=0.02, \mathrm{r}=$ $-0.44)$. The relationships of triglycerides with fat area and fat proportion were significant $(\mathrm{P}$ $=0.05$ and 0.01$)$, as well as that between cholesterol and intima/media area ratio $(\mathrm{P}=$ 0.02 ), but with low coefficients ( $r=0.28,0.35$, and 0.32 , respectively). All other correlations were nonsignificant and weak.

\section{Discussion}

Although a nonsignificant increase in intimal area and thinning of the media created a positive association between LDL and the intima/media ratio, the main finding in this study was that intimal area, percent fat plaque content and media area in the coronary arteries of patients who died from atherosclerosis were virtually independent of their serum lipid levels.

This study was not designed to challenge the well-established knowledge that patients with hypercholesterolemia have an increased possibility of developing atherosclerosis. On the other hand, our results show that, once persons with normal cholesterol levels present the disease in their coronary arteries, their disease will be similar, concerning fat content, to that occurring in persons with high cholesterol.

\section{Methodological considerations and comparison with similar studies}

The relation between serum cholesterol and triglycerides measured during life and autopsy coronary findings has been studied (8), showing that there were fewer branches with severe obstruction in the group with normal cholesterol $(\leq 250 \mathrm{mg} / \mathrm{dL})$ and triglycerides $(\leq 170 \mathrm{mg} / \mathrm{dL})$, and a correlation of the number of segments with severe narrowing $(\geq 75 \%)$ with triglyceride levels. Nevertheless, plaque composition was not evaluated in the cited study (8).

In another investigation (9), total cholesterol and HDL were quantified post mortem in cases of sudden death. More vulnerable plaques (i.e., plaques containing more fat) were associated with an elevated total cholesterol/HDL ratio. Besides verifying lipid levels only $19 \mathrm{~h}$ after death, on average, this study (9), like most other previously published semiquantitative or quantitative studies on plaque composition, stained coronary segments with hematoxylin and eosin or by the Movat method $(10,11)$ employing usual histological processing, which involves embedding in alcohol and xylene. The evaluation of fat content may be hindered due to the extraction of fat promoted by these solvents. Thus, only the "negative image" of fat was taken into account in these studies. If, as is often the case, lipids were interacting with other plaque components, such as proteoglycans and collagen, they would not be demonstrated by the negative image, although present in the area. Furthermore, small artifactual holes could be regarded as areas of removed fat. In contrast, we used frozen histological sections stained with Sudan-IV (a specific method for revealing lipids). In addition, we measured fat by a specific detection method for computer analysis. Therefore, we did not omit small, isolated areas of lipid droplets that could be missed or very difficult to measure if the drawing mode of computer morphometry had been used instead of the detecting mode. A similar staining method was also used for the determination of the effects of a statin on the composition of atherosclerotic plaques, but cholesterol was elevated in both the tested and placebo groups (12).

\section{Mechanisms of atherosclerosis}

The similarities in plaque composition observed in patients with low and high lipids suggest that, besides serum lipid levels, other factors influence the fat content of atherosclerotic lesions. In another study by our group, we already suggested that atherosclerotic plaque development, or its prevention, could occur independently of plasma cho- 
lesterol. Studying the effects of red wine in rabbits submitted to a hypercholesterolemic diet, we have documented the efficacy of red wine against atherosclerotic plaque development in spite of very high concentrations of plasma cholesterol (13). This difference between cholesterol levels and the intensity of atherosclerosis was also detected by comparing two different strains of transgenic mice, C57BL/6J and $\mathrm{C} 3 \mathrm{H} / \mathrm{HeJ}$ (14). While on the atherogenic diet, both had increased levels of cholesterol, triglycerides, Lp(a) lipoprotein, cholesterol ester transfer protein, apolipoprotein A and other molecules, but did not differ in the amount of these lipids in the liver; however, only the first strain developed fatty streaks. The differences found between the two strains involve other substances, including hepatic oxidized lipids.

The different influences on the development of atherosclerotic plaque may be a combination of inflammation/infection, endothelial dysfunction, oxidative stress, adhesion molecules, and genetic factors. However, the extent to which these were operative in our study was not assessed.

\section{Modes of presentation of ischemic heart disease}

Differences in plaque composition have been described according to the type of manifestation of coronary disease $(15,16)$. Cases with unstable angina and acute myocardial infarction showed more pultaceous debris in the presence of severe obstruction than in the presence of moderate obstruction, while this variation was not seen in sudden death. The study of the relation between after-death quantification of serum lipids and plaque content cited above (9) focused on sudden death. On the other hand, our patients had established coronary disease, most of them presenting myocardial infarcts (either recent and/or old) or submitted to a coronary procedure. No case of sudden death was included. Thus, at least part of the disagreement in results (the authors found plaques containing more fat in association with a higher total cholesterol/HDL ratio) between their study and ours may be attributed to the distinct characteristics of the study population. Some caution is required in the extrapolation of our results to cases of sudden death.

\section{Clinical implications}

Clinical manifestations of atherosclerotic disease, which are dependent on plaque size (17), characteristics (18), and degree of stenosis (19), and not on risk factors, are possibly alike in the two groups; therefore, the types of plaques ("soft" or "hard"), and their possible implications, such as plaque rupture, cannot be inferred based on serum lipid levels in a patient with diagnosed coronary atherosclerosis. Hence, alternative indices that can reflect atherosclerotic disease are needed. They could be inflammatory or thrombogenic factors or others. Endothelial dysfunction may also indicate early atherosclerosis and is of prognostic importance.

Patients with hypercholesterolemia benefit from cholesterol-lowering therapy (4), a fact interpreted as a consequence of a decrease in fat content of the atherosclerotic plaques and thus of their stabilization. However, patients with normal cholesterol also show reduced ischemic events when treated with statins $(20,21)$. These results were interpreted as consequences of other types of mechanisms involved in atherosclerosis, such as anti-thrombotic, anti-inflammatory mechanisms and interference with nitric oxide synthase activity (22).

Our results indicate that in patients with normal cholesterol (and fractions) part of the effects of these drugs could be the same as in those with hypercholesterolemia, including effects directly related to a decrease in lipid plaque content. It is possible that even patients with normal cholesterol levels could benefit from further reduction of their cholesterol levels. 


\section{Acknowledgments}

The authors are grateful to Cândido de Oliveira Gonçalves for help in dissecting the coronary arteries, to Solange Aparecida Consorti for technical support in the preparation of Sudan-stained slides, and to Gerson Lilá Ramos for help with the data bank.

\section{References}

1. Linton MF, Fazio S. A practical approach to risk assessment to prevent coronary artery disease and its complications. Am J Cardiol 2003; 92: 19i-26i.

2. LaRosa JC, He J, Vupputuri S. Effect of statins on risk of coronary disease: a meta-analysis of randomized controlled trials. JAMA 1999; 282: 2340-2346.

3. Lee YS, Chang HJ, Kim SH, Chung YR, Hong DH, Na HW, et al. Risk factor profile of coronary artery disease in patients with low cholesterol level. J Am Coll Cardiol 2000; 35 (Suppl A): 309A.

4. The Scandinavian Simvastatin Survival Study Group. Randomised trial of cholesterol lowering in 4444 patients with coronary heart disease: the Scandinavian Simvastatin Survival Study (4S). Lancet 1994; 344: 1383-1389.

5. Davies MJ, Richardson PD, Woolf N, Katz DR, Mann J. Risk of thrombosis in human atherosclerotic plaques: role of extracellular lipid, macrophage, and smooth muscle cell content. Br Heart J 1993; 69: $377-381$.

6. Fernandez-Ortiz A, Badimon JJ, Falk E, Fuster V, Meyer B, Mailhac $A$, et al. Characterization of the relative thrombogenicity of atherosclerotic plaque components: implications for consequences of plaque rupture. J Am Coll Cardiol 1994; 23: 1562-1569.

7. Friedewald WT, Levy RI, Fredrickson DS. Estimation of the concentration of low-density lipoprotein cholesterol in plasma, without use of the preparative ultracentrifuge. Clin Chem 1972; 18: 499-502.

8. Cabin HS, Roberts WC. Relation of serum total cholesterol and triglyceride levels to the amount and extent of coronary arterial narrowing by atherosclerotic plaque in coronary heart disease. Quantitative analysis of 2,037 five $\mathrm{mm}$ segments of 160 major epicardial coronary arteries in 40 necropsy patients. Am J Med 1982; 73: 227-234.

9. Burke AP, Farb A, Malcom GT, Liang YH, Smialek J, Virmani R. Coronary risk factors and plaque morphology in men with coronary disease who died suddenly. N Engl J Med 1997; 336: 1276-1282.

10. Mautner SL, Lin F, Mautner GC, Roberts WC. Comparison in women versus men of composition of atherosclerotic plaques in native coronary arteries and in saphenous veins used as aortocoronary conduits. J Am Coll Cardiol 1993; 21: 1312-1318.

11. Varghese PJ, Arumugam SB, Cherian KM, Walley V, Farb A, Virmani R. Atheromatous plaque reflects serum total cholesterol levels: a comparative morphologic study of endarterectomy coronary atherosclerotic plaques removed from patients from the southern part of India and Caucasians from Ottawa, Canada. Clin Cardiol 1998; 21 :
335-340.

12. Crisby M, Nordin-Fredriksson G, Shah PK, Yano J, Zhu J, Nilsson J. Pravastatin treatment increases collagen content and decreases lipid content, inflammation, metalloproteinases, and cell death in human carotid plaques: implications for plaque stabilization. Circulation 2001; 103: 926-933.

13. da Luz PL, Serrano Junior CV, Chacra AP, Monteiro HP, Yoshida VM, Furtado $M$, et al. The effect of red wine on experimental atherosclerosis: lipid-independent protection. Exp Mol Pathol 1999; 65: 150-159.

14. Liao F, Andalibi A, deBeer FC, Fogelman AM, Lusis AJ. Genetic control of inflammatory gene induction and NF-kappa B-like transcription factor activation in response to an atherogenic diet in mice. $J$ Clin Invest 1993; 91: 2572-2579.

15. Kragel AH, Reddy SG, Wittes JT, Roberts WC. Morphometric analysis of the composition of coronary arterial plaques in isolated unstable angina pectoris with pain at rest. Am J Cardiol 1990; 66: 562567.

16. Kragel AH, Reddy SG, Wittes JT, Roberts WC. Morphometric analysis of the composition of atherosclerotic plaques in the four major epicardial coronary arteries in acute myocardial infarction and in sudden coronary death. Circulation 1989; 80: 1747-1756.

17. Bezerra HG, Higuchi ML, Gutierrez PS, Palomino SA, Silvestre JM, Libby $\mathrm{P}$, et al. Atheromas that cause fatal thrombosis are usually large and frequently accompanied by vessel enlargement. Cardiovasc Pathol 2001; 10: 189-196.

18. Libby P. Current concepts of the pathogenesis of the acute coronary syndromes. Circulation 2001; 104: 365-372.

19. Qiao JH, Fishbein MC. The severity of coronary atherosclerosis at sites of plaque rupture with occlusive thrombosis. J Am Coll Cardiol 1991; 17: 1138-1142.

20. Schwartz GG, Olsson AG, Ezekowitz MD, Ganz P, Oliver MF, Waters $D$, et al. Effects of atorvastatin on early recurrent ischemic events in acute coronary syndromes: the MIRACL study: a randomized controlled trial. JAMA 2001; 285: 1711-1718.

21. Heart Protection Study Collaborative Group. MRC/BHF Heart Protection Study of cholesterol lowering with simvastatin in 20,536 high-risk individuals: a randomised placebo-controlled trial. Lancet 2002; 360: 7-22.

22. Libby $\mathrm{P}$, Aikawa M. Mechanisms of plaque stabilization with statins. Am J Cardiol 2003; 91 (4A): 4B-8B. 